\title{
Der Einfluss des lokalen Hochschulangebots auf die Studienwahl
}

\author{
Stefan Denzler $\cdot$ Stefan C. Wolter
}

\begin{abstract}
Zusammenfassung: Verschiedene Studien belegen, dass das lokale Hochschulangebot bzw. die Distanz zur nächstgelegenen Universität das Studienverhalten beeinflusst: Die Wahrscheinlichkeit, ein Studium zu ergreifen, steigt mit der geographischen Nähe zu einer Hochschule. In der vorliegenden Studie wird die Frage auf die Wahl des Studienfachs sowie der Hochschulinstitution ausgedehnt und in einem humankapitaltheoretischen Ansatz am Beispiel der ETH Zürich, der Universität Luzern sowie der pädagogischen Hochschulen analysiert. Auf der Basis einer repräsentativen Maturandenbefragung in der deutschsprachigen Schweiz $(n=1454)$ kann regressionsanalytisch gezeigt werden, dass die Distanz zur nächstgelegenen Hochschule auch einen Einfluss auf die Fächer- und die Institutionenwahl hat. Die Befunde sind hochschulpolitisch von Bedeutung, weil sie Hinweise auf eine angebotsinduzierte Studiennachfrage liefern. Zudem zeigen die Ergebnisse, dass bei Studierenden mit höherem sozioökonomischen Status das Studienverhalten durch die Distanz nicht beeinflusst wird, was als Indiz dafür gewertet werden kann, dass die Bedeutung der Entfernung einer Hochschule in Wirklichkeit auf Unterschieden in den Kosten eines Studiums gründet.
\end{abstract}

Schlüsselwörter: Geographische Distanz zu einer Hochschule $\cdot$ Studienfachwahl · Hochschulangebot · Hochschulsystem · Universität · Pädagogische Hochschule

\section{The influence of local higher education provision on study choice}

\begin{abstract}
Various studies show that local higher education provision and the distance to the nearest university effect study behavior. The probability of university participation increases with the geographical proximity of an institution of higher education. In this study we extend the question to the choice of subject of study and the specific institution using a human capital approach with reference to the ETH Zurich, Lucerne University and teacher training colleges. On the basis
\end{abstract}

\footnotetext{
Online publiziert: 03.11 .2010

(C) VS Verlag für Sozialwissenschaften 2010

S. Denzler $(\bowtie)$

Schweizerische Koordinationsstelle für Bildungsforschung (SKBF);

Universität Lausanne, Entfelderstrasse 61, 5000 Aarau, Schweiz

E-Mail: stefan.denzler@skbf-csre.ch

Direktor Prof. Dr. S. C. Wolter

Schweizerische Koordinationsstelle für Bildungsforschung (SKBF);

Universität Bern; CESifo \& IZA,

Schanzeneckstrasse 1, 3001 Bern, Schweiz

E-Mail: stefan.wolter@vwi.unibe.ch
} 
of a representative survey of holders $(n=1454)$ of the higher education entrance qualification in German-language Switzerland, we show through regression analysis that the distance to the nearest university also has an effect on choice of subject and institution. The results are of policy relevance because they indicate a provision-induced study demand. Furthermore, they show that study behavior is not influenced by distance for students with a high socio-economic status. This can be understood as an indication that the importance of the proximity of a university is actually founded in the difference in study costs.

Keywords: Geographic distance to a university $\cdot$ Higher education provision $\cdot$ Higher education system $\cdot$ Subject choice $\cdot$ Teacher training college $\cdot$ University

\section{Einleitung}

Die Erhöhung der Mobilität im Hochschulsystem ist ein erklärtes Ziel nationaler wie internationaler Hochschulpolitik (OECD 1998; SBF 2004). Nur eine hohe Mobilität zwischen den Hochschulen ermöglicht die bestmögliche Zuordnung von Studierenden und Universitäten (allokative Effizienz). Der dadurch gesteigerte Wettbewerb unter den Hochschulen wird, so wird argumentiert, letztlich auch die Qualität im System erhöhen (produktive Effizienz). In diesem Wettbewerb um talentierte Studierende (Mechtenberg 2005) ist es entscheidend, dass unterschiedlich begabte Studierende an den für ihre Voraussetzungen geeignetsten Hochschulen studieren und dass sie jene Fächer wählen können, die ihren Neigungen und Fähigkeiten am besten entsprechen. Begabte junge Menschen sollten nicht aus finanziellen Gründen auf ein Studium verzichten müssen.

Der Umstand, dass in vielen Ländern der überwiegende Teil der Studierenden einer Hochschule aus dem näheren geographischen Umfeld stammt, lässt aber daran zweifeln, ob die Mobilität der Studierenden tatsächlich genügend groß ist, um eine optimale produktive und allokative Effizienz im Hochschulsystem zu erzielen. Wenn die Chancen des Hochschulzugangs aufgrund der unterschiedlichen geographischen Mobilität ungleich verteilt sind, wird das Humankapitalpotenzial nicht ausgeschöpft, was die Wirksamkeit des gesamten Bildungssystems beeinträchtigt. Die unterschiedliche Erreichbarkeit von Hochschulen tangiert aber auch die Frage der Chancengerechtigkeit (vgl. Turley 2009). Die geographische Dichte des Hochschulangebots und die Sensitivität der individuellen Nachfrage nach einem Studium in Abhängigkeit von der Distanz zwischen dem Wohnort und der nächstgelegenen Hochschule stellen daher für die Bildungspolitik wichtige Größen dar.

Der Zusammenhang zwischen der Distanz zur nächstgelegenen Hochschule und der Studierneigung ist in der empirischen Literatur seit langem und relativ gut belegt (vgl. bspw. Tinto 1973; McHugh und Morgan 1984; Sà et al. 2004; Avery und Hoxby 2004; Long 2004; Drewes und Michael 2006; Frenette 2006, 2009; Turley 2009). Trotzdem ist nicht eindeutig klar, ob dieser Zusammenhang auch wirklich kausaler Natur ist, denn die Schätzung der Korrelation könnte durch das Vorliegen von Endogenität verzerrt sein. Es ist nämlich nicht auszuschließen, dass die Wohnortwahl endogen ist, also durch andere, unbeobachtete Faktoren, welche auch einen Einfluss auf die Studierneigung haben - wie etwa die soziale Herkunft - beeinflusst wird. So ist anzunehmen, dass bildungsfernere Schichten tendenziell weiter von einem urbanen Zentrum und somit weiter von einer 
Hochschule entfernt wohnen als bildungsnahe Personen (Coradi Vellacott 2007). Der Grund für die geringere Studierneigung wäre somit eher im sozialen und Bildungshintergrund als in der Distanz zu einer Hochschule zu suchen. Andererseits unterliegt der Zugang zu den Hochschulen in den meisten Ländern Restriktionen in Form von Eintrittstests und Studiengebühren, die von Hochschule zu Hochschule stark variieren können. Zur Selbstselektion der Studierenden tritt also noch die Fremdselektion durch die Hochschulen hinzu. Beide Aspekte sind mögliche Quellen von Verzerrungen bei der Schätzung des zu untersuchenden Zusammenhangs.

Im folgenden Beitrag wird deshalb eine empirische Neubeurteilung des Effekts der Distanz auf den Studienentscheid vorgenommen, die möglichst frei von Endogenität und unbeobachtbaren Selektionsentscheiden sein soll und gleichzeitig auf adäquaten, der Fragestellung angepassten Daten beruht. Dabei sind folgende Punkte hervorzuheben:

Erstens werden nicht die Auswirkungen der Distanz auf die Studierneigung an sich - also auf den Entscheid, ein Studium aufzunehmen oder nicht - untersucht, sondern die Entscheide bezüglich des gewählten Studienfachs und der präferierten Hochschule. Zudem wird die Studienwahl nur zwischen jenen Maturanden verglichen, die sich für ein Studium auf der Tertiärstufe entschieden haben. Dies hat den Vorteil, dass endogene Faktoren, die mit der generellen Studierneigung zusammenhängen können (bspw. Bildungsferne oder Bildungserwartungen der Eltern oder der Einfluss der lokalen Peers) kaum ins Gewicht fallen. Es scheint außerdem sinnvoll, anstelle der allgemeinen Studierneigung nur die Fächer- und Institutionenwahl zu betrachten, weil die Hochschul-Übertrittsquote in der Schweiz mit etwa $90 \%$ sehr hoch ist. ${ }^{1}$

Zweitens eignet sich die Schweiz für die Untersuchung dieser Fragen auch deshalb, weil Maturanden eine Studienberechtigung für alle Hochschulen und alle Fächer besitzen (mit der Ausnahme von Medizin in der deutschen Schweiz). Sowohl die Wahl der Hochschule respektive des Hochschultyps als auch die des Studienfachs sind individuelle Entscheide, die keinerlei Restriktionen seitens der Hochschulen unterliegen. Weiter verlangen alle Hochschulen eher geringe und im Allgemeinen ähnlich hohe Studiengebühren, die somit keinen Einfluss auf die Wahl des Studienortes haben sollten.

Drittens erlaubt der hier verwendete Datensatz den Einbezug einer großen Zahl von Kontrollvariablen zu persönlichen Merkmalen. Dies ermöglicht es, alternative Erklärungen zum Distanzeffekt besser abzubilden und zu kontrollieren.

Viertens ist die Schweiz zwar geographisch kleinräumig und mit öffentlichen Verkehrsmitteln gut vernetzt, aber die Reisedistanzen können aufgrund der Topographie doch erheblich sein. Deshalb wird in diesem Aufsatz mit den tatsächlichen Reisezeiten operiert und nicht, wie in der Literatur üblich, mit den weniger aussagekräftigen Luftliniendistanzen.

Der Aufsatz ist wie folgt gegliedert: Zuerst werden die theoretischen Ansätze vorgestellt, die den Einfluss des lokalen Hochschulangebots respektive Distanzeffekte auf den Studienentscheid erklären, und es wird die vorhandene empirische Literatur zusammengefasst. Anschließend werden die Forschungsfragen präzisiert und die Arbeitshypothesen entwickelt. Nach einem kurzen Exkurs zum schweizerischen Hochschulsystem wird das methodische Vorgehen erläutert; danach werden die Ergebnisse dargestellt und besprochen. Ein abschließendes Fazit versucht, eine vorläufige Bilanz zu ziehen. 


\section{Der Einfluss der Distanz zur Hochschule auf Studienentscheide}

\subsection{Theoretische Überlegungen}

Verschiedene Faktoren können erklären, weshalb die geographische Distanz zur nächstgelegenen Hochschule einen Einfluss auf das Studienverhalten hat und insbesondere auf die Wahrscheinlichkeit, überhaupt zu studieren. Die möglichen Erklärungen lassen sich vier Kategorien zuordnen:

a) Sozialräumliche Segregation. Die Wohnortwahl ist auf ein bestimmtes Selektionsverhalten zurückzuführen. Bildungsferne Schichten wohnen tendenziell eher in peripher gelegenen und damit in von Hochschulen entfernter gelegenen Gebieten (vgl. Kesselring 1979; Salvi et al. 2004; Coradi Vellacott 2007) und können deshalb unabhängig von der Distanz zu einer Hochschule eine geringere Studierneigung aufweisen. ${ }^{2}$

b) Peer-Effekte/Nachbarschaftseffekte. Ähnliche Personengruppen (bspw. Akademiker, ungelernte Arbeiter, Migranten usw.) wohnen an ähnlichen und von Hochschulen ähnlich weit entfernten Orten; sie beeinflussen sich gegenseitig in ihrem Studierverhalten (Fabel et al. 2002, S. 512; Spieß und Wrohlich 2010, S. 472). Auch dürften sogenannte Spillover-Effekte dabei eine Rolle spielen, indem das lokale Hochschulangebot das Studienverhalten der Umgebung beeinflusst. So dürften Personen, die in der Nähe einer Universität aufwachsen, ein Hochschulstudium eher als den üblichen Ausbildungsweg betrachten als Personen in abgelegenen Gebieten (Chau 2004, S. 249).

c) Emotionale Kosten. Das Wegziehen von zu Hause ist mit emotionalen Kosten verbunden. Personen sind in ihrem lokalen Umfeld verwurzelt (Familie, Freunde, soziale Netzwerke, Vereine usw.) und wollen dieses nicht verlassen (Spieß und Wrohlich 2010, S. 471).

d) Transaktionskosten. Humankapitaltheoretisch betrachtet bedeutet eine größere Distanz zur Hochschule höhere Studienkosten (Fabel et al. 2002). ${ }^{3}$ Dabei fallen einerseits direkte Kosten etwa in Form von Reise- oder Wohnkosten an, andererseits indirekte Kosten, namentlich Opportunitätskosten wie etwa die entgangenen Ersparnisse, welche durch das billigere Wohnen im elterlichen Haushalt anfallen würden, oder auch die durch das Pendeln entgangene Zeit (Spieß und Wrohlich 2010, S. 471).

Die ersten beiden Erklärungsansätze (a und b) sind so zu interpretieren, dass es nicht die Distanz zur Hochschule ist, welche das Studierverhalten beeinflusst, sondern dass es andere Faktoren sind, die sich gleichzeitig auf das Studierverhalten wie auf die Wahl des Wohnorts auswirken. Der nicht-kausale Bezug der Distanz zum Studierverhalten müsste sich dahingehend äußern, dass eine bestimmte Person, je nach Distanz zur Hochschule, kein anderes Studierverhalten zeigen würde. Bei den letzteren Erklärungsansätzen (c und d) hätte die Distanz zur Hochschule einen kausalen Einfluss auf das Studierverhalten, sei es aus monetären, sei es aus nicht-monetären Gründen.

Um herauszufinden, ob es sich bei der Distanz zur Hochschule um einen kausalen Einfluss auf das Studierverhalten handelt, kann man entweder versuchen, die Selektionseffekte über Kontrollvariablen zu neutralisieren, oder andererseits eine Fragestellung wählen, die von der Wohnortswahl nicht beeinflusst werden dürfte. Selektionseffekte sollten dann keine Rolle mehr spielen. 


\subsection{Ergebnisse aus der empirischen Literatur}

Distanzeffekte hinsichtlich des Studierverhaltens wurden ursprünglich im Zusammenhang mit US-amerikanischen Studien zur innerstaatlichen Migration von College-Studenten untersucht. In den verwendeten Modellen wurde das lokale Hochschulangebot respektive die Distanz berücksichtigt, die mit der Wahl einer Hochschule in einem anderen Bundesstaat verbunden war (vgl. bspw. Tuckman 1970; McHugh und Morgan 1984; Mixon 1992; Mixon und Hsing 1994). Wer näher bei einer Hochschule wohnt, hat eine höhere Wahrscheinlichkeit, sich dort auch einzuschreiben. Die meisten Arbeiten dokumentieren negative Distanzeffekte (Sà et al. 2004, S. 5). Jüngere, auf genaueren Distanzangaben basierende Studien bestätigten diese Befunde. In der Regel ließ sich ein statistisch negativer Einfluss der Distanz auf die Studierneigung empirisch bestätigen (vgl. Kyung 1996; McHugh und Morgan 1984; Avery und Hoxby 2004; Long 2004; Frenette 2006; Alm und Winters 2009). Die in einem Teil dieser Aufsätze überprüfte Annahme, dass sich schulische Fähigkeiten geographisch unabhängig verteilen, oder auch die Beobachtung, dass sozial privilegierte Studierende keinen Distanzeffekten unterliegen, werden dabei häufig als Argumente dafür verwendet, dass es sich beim Distanzeinfluss tatsächlich um einen kausalen Kosteneffekt und weniger um einen Selektionseffekt handelt (Spieß und Wrohlich 2010). Allerdings kann beiden Argumenten widersprochen werden, denn sie vermögen den Einfluss anderer unbeobachteter Faktoren nicht wirklich auszuschließen. So kann die Studierneigung mit Erwartungshaltungen zusammenhängen, die mit der sozialen Herkunft verknüpft sind, und diese kann wiederum die Wohnortswahl bestimmen, sodass selbst bei gleichen schulischen Leistungen die Wahrscheinlichkeit, ein Studium zu ergreifen, nicht von der Distanz zur Universität abhängt, sondern von der sozialen Herkunft und anderen, schwer kontrollierbaren Einflüssen. Das Vorliegen von Endogenität kann eigentlich nur ausgeschlossen werden, wenn das lokale Hochschulangebot oder die Wohnorte exogen variiert werden. Eine solche exogene Variation, die sozusagen einem natürlichen Experiment entspricht, machten sich nun beispielsweise Frenette (2009), aber auch Currie und Moretti (2003) zunutze, indem sie die Effekte von Neugründungen von Universitäten analysierten. Wäre der Einfluss der Distanz nicht kausal, dann dürfte die Verfügbarkeit eines neuen Hochschulangebots in einer Region, die bislang in größerer Distanz zu anderen Hochschulen lag, die Studierwahrscheinlichkeit in dieser Region nicht beeinflussen. Wenn das neue Hochschulangebot aber einen positiven Einfluss auf die Studierendenquoten in der betroffenen Region ausübt, so muss von einem kausalen Einfluss der Distanz ausgegangen werden.

Ein wiederum etwas anderer Ansatz besteht darin, nicht die Studierneigung an sich zu untersuchen, sondern die Wahl einer bestimmten Hochschule respektive eines Hochschultyps oder eines bestimmten Studienfachs. In diesem Sinne verfahren (Griffith und Rothstein 2009), wenn sie die Bewerbungen bei sogenannt „selektiven“ Colleges untersuchen und feststellen, dass die Wahrscheinlichkeit, sich bei einem selektiven College für einen Studienplatz zu bewerben, mit der Distanz abnimmt. Diese Ergebnisse erhärten die Hypothese, dass es sich bei der Distanz letztlich um Kostenfaktoren handelt, die einen kausalen Einfluss auf das Studierverhalten haben (Avery und Hoxby 2004). 


\subsection{Forschungsfragen und Arbeitshypothesen}

Im vorliegenden Aufsatz kombinieren wir zwei der oben geschilderten Analysemethoden. Zum einen wird der Distanzeffekt in Bezug auf eine spezifische Fächer- respektive Institutionenwahl untersucht, zum anderen untersuchen wir diesen Effekt am Beispiel einer Hochschulneugründung. Dabei geht es in beiden Fällen nicht darum zu untersuchen, ob die Studierneigung durch die Distanz zu einer Hochschule beeinflusst wird, sondern ob die Wahl des Studienfachs oder der Hochschule distanzabhängig ist.

Im ersten Fall untersuchen wir die Wahrscheinlichkeit, an der ETH Zürich (ETHZ) zu studieren. Die ETHZ zeichnet sich unter den übrigen Hochschulen in der deutschen Schweiz nicht nur durch eine hohe internationale Reputation aus, sondern sie bietet auch Fächer (wie etwa das Ingenieur- oder das Architekturstudium) an, die an anderen universitären Hochschulen nicht angeboten werden. Unter der Annahme geographisch gleich verteilter Präferenzen für diese Fächer kann folgende Hypothese aufgestellt werden: Wenn die Distanz zu einer Hochschule keinen kausalen Einfluss auf das Studierverhalten hat, erwarten wir, dass die Wahrscheinlichkeit, ein Studium an der ETHZ anzustreben, von der geographischen Distanz zu dieser unabhängig ist.

Im zweiten Untersuchungsfall wird analysiert, ob die Nähe zu der erst im Jahr 2000 gegründeten Spartenuniversität Luzern die Wahrscheinlichkeit erhöht, ebendiese Fächer zu studieren, die an dieser Universität angeboten werden. Wiederum unter der Annahme, dass die Präferenz für bestimmte Studienfächer grundsätzlich nicht vom lokalen Fächerangebot abhängt, kann auch hier die Hypothese aufgestellt werden, dass bei Abwesenheit von Distanzrestriktionen in allen Regionen ungefähr die gleiche Verteilung in der Studienfächerwahl beobachtet werden müsste. Wären (gewisse) Studierende hingegen Distanzrestriktionen unterworfen, dann würde die Wahrscheinlichkeit, jene Fächer zu studieren, die durch die lokale Universität angeboten werden, mit der Nähe zu dieser Hochschule steigen.

Schließlich untersuchen wir am Beispiel der pädagogischen Hochschulen, inwiefern sich das lokale Angebot auf die Wahl dieses Hochschultyps auswirkt. Die pädagogischen Hochschulen wurden zwischen 2002 und 2005 als dritter Hochschultypus neben den universitären und den Fachhochschulen eingerichtet und bieten seither auf tertiärer Stufe Studiengänge für das Lehramt an. Da die pädagogischen Hochschulen eine größere Angebotsdichte aufweisen als die universitären Hochschulen, haben wir hier, analog zur neu gegründeten Universität Luzern, die Situation einer lokalen Alternative zu einer allenfalls weiter entfernten Universität. Diese lokale Alternative besteht jedoch nicht aus einer universitären Hochschule mit eingeschränktem Fächerangebot (bspw. Jus im Fall der Universität Luzern), sondern aus einem anderen Hochschultypus mit dem Angebot einer berufsspezifischen Ausbildung in einem einzigen Berufsfeld, nämlich dem Lehrberuf. Es stellt sich damit die Frage, ob sich auch in diesem Fall Hinweise auf ein angebotsinduziertes Nachfrageverhalten finden lassen. Gemäß den oben ausgeführten Hypothesen ist zu vermuten, dass die Wahrscheinlichkeit, ein Studium an einer pädagogischen Hochschule aufzunehmen, mit zunehmender Distanz zur nächsten Universität steigt. 


\section{Hochschullandschaft und Zugang zur Hochschule in der Schweiz}

Inhaber einer gymnasialen Maturität (Abschlussexamen der allgemeinbildenden Sekundarstufe II) haben in der Schweiz freien Zugang zu den Studiengängen auf Hochschulstufe (mit Ausnahme der Medizin). Die große Mehrheit der Gymnasiumsabsolventen (etwa zwei Drittel) wählen ein universitäres Studium an einer kantonalen Universität oder an einer der beiden eidgenössischen technischen Hochschulen (ETH) in Zürich und Lausanne. ${ }^{4}$ Ein kleinerer Anteil (ein gutes Viertel) entscheidet sich für eine Fachhochschulausbildung oder für einen Studiengang an einer pädagogischen Hochschule. Für die meisten Maturanden, die ein Studium an einer Fachhochschule aufnehmen, liegt der Grund dafür in der Tatsache, dass diese in den Bereichen Kunst/Gesundheit und Soziales Studiengänge anbieten, welche an den akademischen Universitäten nicht angeboten werden. Insgesamt setzen über $90 \%$ der Maturanden ihre Bildungslaufbahn mit einer Hochschulausbildung fort. ${ }^{5}$ Diese im internationalen Vergleich hohe Übertrittsquote erklärt sich unter anderem auch mit der strengeren Selektion der Schülerinnen und Schüler durch die Gymnasien. Derzeit liegt die Maturandenquote bei rund 20\% eines Jahrgangs (vgl. SKBF 2010).

\subsection{Struktur der schweizerischen Hochschullandschaft}

Die Schweiz verfügt über zehn kantonale Universitäten, wovon drei (Luzern, St. Gallen, Tessin) nicht Universitas litterarum im eigentlichen Sinn sind, also nicht den gesamten Fächerkanon einer Universität anbieten, sondern sich auf zwei, drei Fachgebiete spezialisieren. Hinzu kommen die zwei eidgenössischen technischen Hochschulen mit ihrem Angebot an ausgeprägt technisch-naturwissenschaftlichen Fächern, die zum Teil nur an diesen beiden Hochschulen angeboten werden, zum Teil aber auch an Universitäten studiert werden können. Den beiden ETH mit ihren Standorten in der Deutschschweiz und in der Westschweiz kommt auch deshalb eine besondere Bedeutung zu, weil sie beide über eine international herausragende Reputation verfügen. ${ }^{6}$

Bei den drei Spartenuniversitäten Lugano ${ }^{7}$, Luzern und St. Gallen handelt es sich in den ersten beiden Fällen um Neugründungen und im Falle von St. Gallen ${ }^{8}$ um eine Universität, welche aus einer Handelsakademie hervorgegangen ist (gegründet im 19. Jahrhundert, später Handelshochschule mit Schwerpunkt Betriebswirtschaftslehre). Für unsere Arbeit ist die neugegründete Universität Luzern von Interesse, da sie erst seit 2000 besteht und bislang eine sehr starke Konzentration auf eine einzige Fakultät (Rechtswissenschaften) aufweist. Die Maturanden, deren Studienwahl in diesem Aufsatz untersucht wird, sind also alle vor der Gründung dieser Universität geboren worden, und bei der relativ geringen Mobilität der Schweizer Bevölkerung kann praktisch ausgeschlossen werden, dass die Wohnortswahl der Eltern durch die Gründung dieser Universität und ihre Spezialisierung auf das Fach Jurisprudenz beeinflusst wurde.

\subsection{Finanzielle Zugangsbarrieren}

Auch wenn es auf institutioneller Seite für Gymnasiumsabsolventen praktisch keine Hindernisse beim Zugang zum Hochschulstudium gibt und die Studiengebühren in der 
Deutschschweiz mit durchschnittlich knapp 1500 Franken pro Jahr (SKBF 2010, S. 190)9 nicht sehr hoch sind, bedeutet dies nicht, dass alle potenziellen Studierenden ihre Entscheide völlig frei von finanziellen Restriktionen treffen können. In der Schweiz sind es vor allem die Lebenshaltungskosten, die einen Studien(-orts-)entscheid stark beeinflussen können. Die meisten Universitäten befinden sich in Städten mit hohem Preisniveau und vor allem hohen Wohnkosten.

Im Elternhaus wohnende Studierende benötigen nach einer Erhebung des Bundesamts für Statistik (s. BFS 2005, 2007, 2009) durchschnittlich 1300 Franken monatlich, um den Lebensunterhalt zu bestreiten (inkl. Studiengebühren usw.). Diese Lebenshaltungskosten steigen für außerhalb des Elternhauses wohnende Studierende auf durchschnittlich 1800 Franken. ${ }^{10}$ Schon heute tragen Studierende rund $40 \%$ ihrer Lebenshaltungskosten durch Erwerbsarbeit neben dem Studium (BFS 2005). Die Möglichkeiten der Selbstfinanzierung sind aber durch zwei Faktoren begrenzt: Einerseits hängen die Arbeitsmöglichkeiten für Studierende stark von der wirtschaftlichen Situation ab, andererseits schränken die immer stärker strukturierten Studiengänge eine freie Zeiteinteilung und somit die Nebenerwerbsmöglichkeiten der Studierenden stark ein. Die Möglichkeiten der Fremdfinanzierung sind in der Schweiz ebenfalls relativ eingeschränkt, da auf der einen Seite Stipendien nur an Studierende vergeben werden, deren Eltern praktisch kein Vermögen haben sowie ein sehr niedriges Einkommen nachweisen, und da es auf der anderen Seite sowohl auf privater wie staatlicher Seite kaum Darlehensmöglichkeiten gibt. Wohl deshalb beziehen heute lediglich 16\% der Studierenden ein Stipendium oder ein Darlehen (BFS 2005, S. 25). ${ }^{11}$

Der Umstand, dass in der Schweiz für ein Studium relativ hohe finanzielle Beiträge der Eltern notwendig sind und dass die Lebenshaltungskosten sich praktisch verdoppeln, wenn man während des Studiums nicht zu Hause wohnen kann, sowie die relativ beschränkten Möglichkeiten, an Stipendien oder Darlehen zu kommen, lässt die Vermutung zu, dass die Wahl des tatsächlichen Studienorts von der geographischen Nähe zu einer Hochschule abhängt und dass diese Wahl zusätzlich von der sozioökonomischen Position der Eltern beeinflusst wird.

\section{Daten und Methode}

\subsection{Daten}

Die empirischen Analysen beruhen auf einer repräsentativen Stichprobe von 1567 Maturanden in neun deutschschweizerischen Kantonen. ${ }^{12}$ Die Schüler wurden kurz vor der Hochschulreife (Maturitätsexamen) zur Studien- und Berufswahl befragt. Die Befragung fand damit zu einem Zeitpunkt statt, in dem der Ausbildungsentscheid der Maturanden konkret ansteht und getroffen werden muss, da sich zu diesem Zeitpunkt viele für das erste Studiensemester an der Universität einschreiben müssen. Die erhobenen Aussagen beziehen sich somit nicht auf eine bereits umgesetzte Studienwahl, sondern auf eine konkrete, direkt anstehende Wahl.

Für die Befragung wurde eine mehrstufige Clusterstichprobe konzipiert, bei der auf Kantonsebene eine systematische Auswahl (bei kleinen Kantonen eine Vollerhebung) 
an Gymnasien erfolgte. Auf der zweiten Stufe, innerhalb der Schulen, wurden einzelne Abschlussklassen zufällig ausgewählt.

Die Datenerhebung wurde im März 2006 mittels schriftlicher Fragebogen klassenweise in den ausgewählten Abschlussklassen durchgeführt. Die Befragung fand nach einheitlichen Kriterien während der regulären Schulzeit unter Aufsicht der Klassenlehrkraft statt. Mit diesem Vorgehen sollten eine möglichst hohe Datenqualität und relativ homogene Klassensamples mit geringer Ausfallquote erzielt werden. ${ }^{13}$ Die bereinigte Gesamtstichprobe umfasst 1454 Beobachtungen.

Wenn im Folgenden Distanzeffekte auf die Fächerwahl oder die Wahl der Institution (Universität oder ETH) untersucht werden, berücksichtigen wir nur Maturanden, die ein universitäres Hochschulstudium anstreben, d. h. an einer der zehn kantonalen Universitäten oder an einer der beiden eidgenössischen technischen Hochschulen (ETH) zu studieren beabsichtigen $(n=933)$. Denn nur bei diesen lässt sich die Studienfachwahl zwischen den Studierenden tatsächlich vergleichen. Im Fall der Wahl einer pädagogischen Hochschule sind hingegen all jene Maturanden in der Analyse berücksichtigt, welche grundsätzlich beabsichtigen, ein Hochschulstudium zu ergreifen, also einen Studiengang an einer universitären, einer Fach- oder einer pädagogischen Hochschule (Tertiär A) anstreben $(n=1339)$.

\subsection{Operationalisierungen}

Die Schülerinnen und Schüler wurden zu ihrem Berufswunsch sowie zu der von ihnen angestrebten Ausbildung befragt. Die Daten enthalten weitere Informationen zur Person (Geschlecht, Alter, familiäre Konstellation, Freizeitaktivitäten), zur sozioökonomischen Herkunft (Bildung, berufliche Stellung und Wohnform der Eltern) sowie zur aktuellen Schulsituation. Die Schwerpunktfächer, die an schweizerischen Gymnasien angeboten werden, wurden nach Profilen zusammengefasst: Sprachen, Mathematik/Naturwissenschaften, Wirtschaft/Recht, musische Fächer (Musik und bildnerisches Gestalten). Ferner wurden die jüngsten Zeugnisnoten in den Hauptfächern erfragt. Die am Klassenmittel standardisierten Notenwerte wurden als Indikatoren für die schulische Leistung verwendet. Von den Noten erweisen sich vor allem die Mathematiknoten als gute Prädiktoren der Fächer- und Hochschulwahl, weshalb die Einflüsse der anderen Noten in der empirischen Auswertung nicht mehr gesondert dargestellt werden. Motive und Präferenzen bei der Studienwahl wurden mittels vorgegebener Items erhoben. Auf der Basis dieser Items wurden später faktoranalytisch verschiedene Skalen zu Konstrukten wie „Wissenschaftsorientierung“" oder „Karriereorientierung“ gebildet.

Zur Bestimmung der Schichtzugehörigkeit wurde eine Variable gebildet, welche anhand der Angaben zum höchsten Bildungsabschluss sowie zur höchsten beruflichen Stellung mindestens eines Elternteil der befragen Maturanden eine Einschätzung der sozioökonomischen Stellung (SES) vornimmt. Dabei wurden die sieben Kategorien der Bildungsvariablen unter Berücksichtigung der beruflichen Stellung vier verschiedenen Schichten (niedriger sozioökonomischer Status bis hoher sozioökonomischer Status) zugewiesen (vgl. Grafik im Anhang). Dieses Vorgehen wurde in Anlehnung an eine vergleichbare Praxis des BFS (vgl. BFS 2009) hauptsächlich deshalb gewählt, weil die Definition der 
Schichtzugehörigkeit mittels nominaler Kriterien erfolgt und nicht anhand eines metrisch skalierten Indexes gebildet werden konnte.

Um zwischen den möglichen Gründen für die Distanzeffekte weiter differenzieren zu können, werden bei den Analysen verschiedene Kontrollvariablen berücksichtigt. Mit den Fächerprofilen an den Gymnasien können beispielsweise gewisse Peer-Effekte kontrolliert werden, da die Wahl des Studienfachs erfahrungsgemäß durch das Schwerpunktfach am Gymnasium und die Studienwahl der Peers am Gymnasium beeinflusst wird. Weiter wird mit zwei klassischen Freizeitaktivitäten, Sport und Pfadfinderei, berücksichtigt, dass es die Einbindung in lokale soziale Netze sein könnte, welche die Hochschulwahl beeinflusst. Denn ein hohes Engagement bei diesen Freizeitaktivitäten geht in der Regel mit einem stärkeren lokalen Eingebundensein einher (vgl. bspw. Heinemann und Horch 1981). Ein solches soziales Netzwerk könnte die Neigung, an einer weiter entfernten Hochschule zu studieren, negativ beeinflussen.

Um den Einfluss der geographischen Nähe des Studienangebots auf die Studienwahl zu untersuchen, wurde für jeden Schüler die Distanz zur nächstgelegenen Universität berechnet, und zwar indem die Zeit ermittelt wurde, die für die kürzeste Verbindung mit öffentlichen Verkehrsmitteln vom Wohnort bis zum Bahnhof des Universitätsstandortes benötigt wird. ${ }^{14}$ Dieses Vorgehen gewährleistet, dass abgelegene Gebiete, die mit dem öffentlichen Verkehr schlecht erschlossen sind, entsprechend richtig erfasst werden, was bei einer Messung der geographischen Distanz (Luftdistanz) nicht der Fall wäre. Reisedistanzen mit anderen Verkehrsmitteln (bspw. Automobil) wurden nicht berechnet, weil der Gebrauch von privaten Verkehrsmitteln unter schweizerischen Studierenden wegen der Kosten und der Parkplatzsituation an den Universitätsstandorten wenig verbreitet ist.

Auf der Basis der individuellen Distanzmaße (Reisezeiten) wurde für die Fragestellung der Spartenuniversität (Universität Luzern) eine zusätzliche Dummy-Variable gebildet, die jeweils das Einzugsgebiet der einzelnen Universitäten kennzeichnet. Bezogen auf die Universität Luzern nimmt der Wert der Dummy-Variablen den Wert 1 an, wenn die Reisedistanz zu dieser Universität die kürzeste ist, und 0, wenn eine andere Universität in kürzerer Zeit zu erreichen ist. Um die Robustheit dieser Variable zu testen, wurden alternativ zwei weitere Dummy-Variablen gebildet, welche größere und kleinere Einzugsgebiete kennzeichnen. Die erste Variable (LU-small) nimmt dann den Wert 1 an, wenn die Universität Luzern mindestens um zehn Minuten (pro Reiseweg) schneller erreichbar ist als jede andere Universität. Die zweite Variable (LU-large) nimmt auch dann noch den Wert 1 an, wenn eine alternative Universität bis maximal zehn Minuten schneller erreichbar ist, d. h. der Besuch der Universität Luzern mit einem zeitlichen Mehraufwand verbunden wäre.

\subsection{Empirisches Modell}

Grundlage der empirischen Analysen ist das folgende Modell, bei dem die Studienwahl y (ein bestimmtes Studienfach, eine bestimmte Hochschule bzw. ein bestimmter Hochschultyp) unter Berücksichtigung einer Reihe weiterer Einflussfaktoren in Beziehung zur Distanz Wohnort-Hochschule respektive zum lokalen Hochschulangebot gesetzt wird:

$$
y_{i}=\beta_{0}+\beta_{1} Z_{i}+\beta_{2} P_{i}+\beta_{3} S_{i}+\beta_{4} M_{i}+\beta_{5} H_{i}+\varepsilon_{i}
$$


Dabei stellt $Z$ einen Vektor dar, der die Distanz zur Hochschule berücksichtigt (Distanzvariable oder Dummy-Variable für ein bestimmtes Hochschul-Einzugsgebiet), $P$ ist ein Vektor, der Merkmale der Person sowie des sozioökonomischen Hintergrunds umfasst, $S$ beinhaltet Variablen zur schulischen Situation (Kontrolle des gymnasialen Profils, was unter anderem auch Peer-Effekte abdecken soll), $M$ umfasst individuelle Motive und Präferenzen, und der Vektor $H$ berücksichtigt Freizeitaktivitäten, welche einen Einfluss sowohl auf die Studienfachwahl als auch auf den Studienort haben könnten (Sport und Pfadfinderei). Diese Regressionsfunktion wird mittels eines Probit-Modells ${ }^{15}$ geschätzt. Für die Ausprägung 1 der abhängigen Variablen $(y=1)$ gilt somit das folgende Wahrscheinlichkeitsmodell (Wooldridge 2003, S. 557):

$$
\operatorname{pr}\left(y=1 \mid x_{i}^{\prime}\right)=\Phi\left(\beta_{0}+\beta_{1} x_{i}^{\prime}\right)
$$

Dabei ist $\Phi$ die standardnormal verteilte kumulierte Verteilungsfunktion; $y=1$ gibt an, dass es sich entweder um eine Person mit Berufswunsch Jurist/in, Lehrer/in handelt oder dass sie an der ETH Zürich studieren möchte. Die verschiedenen in den Hypothesen postulierten Determinanten sind im Vektor $x^{\prime}$ zusammengefasst. Wegen der nichtlinearen Natur des bedingten Erwartungswerts von y kann die Schätzung der Modellparameter aber nicht nach der OLS-Methode erfolgen, sondern es muss nach der Maximum-Likelihood-Methode geschätzt werden (Wooldridge 2003, S. 559). Dadurch können die Koeffizienten nur hinsichtlich Signifikanz und Vorzeichen direkt interpretiert werden. Für eine quantitative Interpretation müssen die vorhergesagten Wahrscheinlichkeiten für einzelne relevante Werte der erklärenden Variablen bestimmt und die marginalen Effekte (MPE) berechnet werden. Dazu wird die Veränderung der Wahrscheinlichkeit bei einer marginalen Veränderung der erklärenden Variable bestimmt. ${ }^{16}$

Es werden drei verschiedene Regressionsmodelle (Probit-Modelle) ${ }^{17}$ geschätzt. Im ersten Fall wird die Wahrscheinlichkeit geschätzt, an der ETH Zürich (ETHZ) studieren zu wollen, wobei hier der Einfluss der Entfernung (in Reisezeit gemessen) zur ETHZ auf den Studienentscheid untersucht wird. Im zweiten Fall wird die Wahrscheinlichkeit, sich für ein Rechtsstudium zu entscheiden, geschätzt. Die hier interessierende Einflussgröße ist die Variable, die angibt, ob die Universität Luzern (mit Spezialisierung auf Jurisprudenz) die nächstgelegene Universität ist. Im dritten Fall schließlich wird die Wahrscheinlichkeit, sich für ein Studium an einer pädagogischen Hochschule zu entscheiden, geschätzt. Dabei wird untersucht, inwiefern die Entfernung zur nächstgelegenen Universität den Entscheid für eine pädagogische Hochschule beeinflusst.

\section{Resultate}

\subsection{Deskriptive Ergebnisse}

Von den 933 Maturanden, die sich zu einem universitären Studium entschließen, wählen durchschnittlich 13\% Rechtswissenschaften. Im Einzugsgebiet der Universität Luzern lässt sich schon rein deskriptiv ein signifikant höherer Anteil an Maturanden mit dem Studienziel Rechtswissenschaft feststellen, nämlich $18 \%$, während es außerhalb Luzerns nur $12 \%$ sind. Für ein Studium der Ingenieur- sowie vieler naturwissenschaftlicher Fächer 
kommt nur die ETHZ infrage. Im Mittel wird die ETHZ von einem Viertel der Maturanden, die sich für ein universitäres Studium entschieden haben, präferiert. Die durchschnittliche Reisedistanz für einen Weg zur nächstgelegenen Universität beträgt eine knappe halbe Stunde, die durchschnittliche Distanz zur ETHZ knapp 50 Minuten; mit anderen Worten, ein Großteil der Studierenden könnte an einer Universität studieren, welche in weniger Reisezeit erreichbar ist als die ETH Zürich.

Von den insgesamt 1339 Maturanden, die sich für einen Studiengang an einer Hochschule (Universität oder Fachhochschule) interessieren, streben gut $8 \%$ ein Studium an einer pädagogischen Hochschule an. Diese stammen mehrheitlich aus peripher gelegenen Wohngebieten: Sie hätten mit durchschnittlich über 50 Minuten Reisezeit einen deutlich längeren Weg zur nächsten Universität zurückzulegen als an die lokal gelegene pädagogische Hochschule. Für die anderen $92 \%$ beträgt die mittlere Reisezeit zur nächst gelegenen Universität nur 41 Minuten pro Weg.

\subsection{Resultate der Regressionen}

\subsubsection{Studium an der ETH Zürich}

Tabelle 1 zeigt, dass sich die Distanz zur ETHZ negativ auf den Entscheid für ein ETHStudium auswirkt. Der Effekt ist signifikant, wenn man das volle Modell mit allen Kontrollvariablen schätzt (Modell 3), und nicht unerheblich: Erhöht sich die Distanz zur ETHZ um eine Standardabweichung, so nimmt die Wahrscheinlichkeit, ein ETH-Studium anzustreben, um gut ein Drittel ab. ${ }^{18}$ Außerdem besteht auf dem 10\%-Niveau Nichtlinearität bezüglich des Distanzeffektes (hier nicht dargestellt). Die sozioökonomische Herkunft (SES) hat keinen generellen Einfluss darauf, ob man ein Studium an der ETHZ aufzunehmen gedenkt; allerdings offenbart die Interaktion der Distanzvariablen mit der sozioökonomischen Herkunft (Modell 4) einen signifikanten Distanzeffekt bei Maturanden aus der untersten und der mittleren sozioökonomischen Schicht: Der Interaktionsterm „SES $1 *$ Distanz“ ist auf dem 10\%-Niveau signifikant $(\mathrm{F}[1 ; 40]=3,9 ; \mathrm{p}<0.10)$; der Interaktionsterm „SES $2 *$ Distanz“ entspricht dem Koeffizienten der Variable „Distanz ETHZ“, da die mittlere SES-Schicht die Referenzkategorie darstellt. Der Koeffizient dieser Variable ist ebenfalls signifikant. Der kombinierte Effekt „Distanz*SES 3“ ist hingegen nicht mehr signifikant von Null verschieden, d. h., bei Maturanden aus der obersten sozioökonomischen Schicht zeigt sich keine signifikante Distanzbarriere mehr. Es sind also vor allem die Angehörigen der Mittelschicht, welche durch die geographische Distanz von einem Studium an der ETHZ abgehalten werden. Dieser Befund deckt sich mit den Ergebnissen von Avery und Hoxby (2004), die eine stärkere Distanzabhängigkeit des Entscheids bei den tieferen sozioökonomischen Schichten beschreiben (a.a.O., S. 268). Dass die unterste sozioökonomische Schicht in unserem Fall nicht stärker als die Mittelschicht vom Distanzeffekt betroffen ist, dürfte mit der Verfügbarkeit von Stipendien zusammenhängen, von welchen die Studierenden aus der Mittelschicht in der Regel nicht profitieren können.

Ferner zeigt sich, dass es vor allem mathematisch begabte Studierende sind, welche ein ETH-Studium anstreben: Maturanden, die leistungsmäßig zum obersten Viertel im Fach Mathematik zählen, haben eine mehr als doppelt so hohe Wahrscheinlichkeit, die ETH 
Tab. 1: Wahrscheinlichkeit Wahl eines Studiums an der ETH Zürich (Probit-Regression)

\begin{tabular}{|c|c|c|c|c|c|c|}
\hline & (Modell 1) & (Modell 2) & (Modell 3) & (Modell 4) & (Modell 5) & $\begin{array}{l}\text { MPE } \\
\text { (Modell 3) }\end{array}$ \\
\hline Distanz ETHZ & $\begin{array}{l}-0,21 \\
(0,17)\end{array}$ & $\begin{array}{l}-0,18 \\
(0,14)\end{array}$ & $\begin{array}{l}-0,28 \\
(0,10)^{* *}\end{array}$ & $\begin{array}{l}-0,29 \\
(0,12)^{*}\end{array}$ & $\begin{array}{l}-0,29 \\
(0,11)^{*}\end{array}$ & $\begin{array}{l}-0,07 \\
(0,03)^{*}\end{array}$ \\
\hline Universitätskanton & $\begin{array}{l}-0,10 \\
(0,11)\end{array}$ & $\begin{array}{l}-0,12 \\
(0,09)\end{array}$ & $\begin{array}{l}-0,07 \\
(0,09)\end{array}$ & & & $\begin{array}{l}-0,02 \\
(0,02)\end{array}$ \\
\hline SES 1: tief (d) & & $\begin{array}{l}0,09 \\
(0,17)\end{array}$ & $\begin{array}{l}0,10 \\
(0,16)\end{array}$ & $\begin{array}{l}0,12 \\
(0,15)\end{array}$ & $\begin{array}{l}0,11 \\
(0,16)\end{array}$ & $\begin{array}{l}0,03 \\
(0,04)\end{array}$ \\
\hline SES 2: mittel (d) & & Referenzka & egorie & & & \\
\hline SES 3: hoch (d) & & $\begin{array}{l}-0,28 \\
(0,17)\end{array}$ & $\begin{array}{l}-0,01 \\
(0,15)\end{array}$ & $\begin{array}{l}0,09 \\
(0,13)\end{array}$ & $\begin{array}{l}-0,01 \\
(0,15)\end{array}$ & $\begin{array}{l}-0,00 \\
(0,04)\end{array}$ \\
\hline $\begin{array}{l}\text { Interaktion SES } \\
1 * \text { Distanz }\end{array}$ & & & & $\begin{array}{l}-0,00 \\
(0,13)\end{array}$ & & \\
\hline $\begin{array}{l}\text { Interaktion SES } \\
3 * \text { Distanz }\end{array}$ & & & & $\begin{array}{l}0,22 \\
(0,18)\end{array}$ & & \\
\hline Alter (zentriert) & & $\begin{array}{l}-0,16 \\
(0,08)^{+}\end{array}$ & $\begin{array}{l}-0,13 \\
(0,09)\end{array}$ & $\begin{array}{l}-0,12 \\
(0,10)\end{array}$ & $\begin{array}{l}-0,12 \\
(0,10)\end{array}$ & $\begin{array}{l}-0,03 \\
(0,02)\end{array}$ \\
\hline Frau (d) & & $\begin{array}{l}-0,91 \\
(0,17)^{* * *}\end{array}$ & $\begin{array}{l}-0,61 \\
(0,13)^{* * *}\end{array}$ & $\begin{array}{l}-0,60 \\
(0,13)^{* * *}\end{array}$ & $\begin{array}{l}-0,60 \\
(0,13)^{* * *}\end{array}$ & $\begin{array}{l}-0,16 \\
(0,03)^{* * *}\end{array}$ \\
\hline \multicolumn{7}{|c|}{ Fächerprofil am Gymnasium: } \\
\hline Sprachen (d) & & & $\begin{array}{l}0,28 \\
(0,20)\end{array}$ & $\begin{array}{l}0,29 \\
(0,22)\end{array}$ & $\begin{array}{l}0,28 \\
(0,21)\end{array}$ & $\begin{array}{l}0,08 \\
(0,06)\end{array}$ \\
\hline Math/Science (d) & & & $\begin{array}{l}1,48 \\
(0,23)^{* * *}\end{array}$ & $\begin{array}{l}1,50 \\
(0,25)^{* * *}\end{array}$ & $\begin{array}{l}1,48 \\
(0,23)^{* * *}\end{array}$ & $\begin{array}{l}0,48 \\
(0,08)^{* * *}\end{array}$ \\
\hline Wirtschaft/Recht (d) & & & Referenzkat & egorie & & \\
\hline Musisch (d) & & & $\begin{array}{l}0,61 \\
(0,26)^{*}\end{array}$ & $\begin{array}{l}0,58 \\
(0,26)^{*}\end{array}$ & $\begin{array}{l}0,59 \\
(0,25)^{*}\end{array}$ & $\begin{array}{l}0,19 \\
(0,09)^{*}\end{array}$ \\
\hline $\begin{array}{l}\text { Note Math., } 4 . \\
\text { Quartil (d) }\end{array}$ & & & $\begin{array}{l}0,59 \\
(0,10)^{* * *}\end{array}$ & $\begin{array}{l}0,60 \\
(0,10)^{* * *}\end{array}$ & $\begin{array}{l}0,62 \\
(0,10)^{* * *}\end{array}$ & $\begin{array}{l}0,18 \\
(0,04)^{* * *}\end{array}$ \\
\hline $\begin{array}{l}\text { Interaktion Math. } \\
\text { note*Distanz }\end{array}$ & & & & & $\begin{array}{l}0,09 \\
(0,10)\end{array}$ & \\
\hline $\begin{array}{l}\text { Wissenschaftsorien- } \\
\text { tierung }\end{array}$ & & & $\begin{array}{l}0,21 \\
(0,06)^{* *}\end{array}$ & $\begin{array}{l}0,20 \\
(0,07)^{* *}\end{array}$ & $\begin{array}{l}0,21 \\
(0,06)^{* *}\end{array}$ & $\begin{array}{l}0,06 \\
(0,02)^{* *}\end{array}$ \\
\hline Karriereorientierung & & & $\begin{array}{l}-0,00 \\
(0,06)\end{array}$ & $\begin{array}{l}-0,00 \\
(0,06)\end{array}$ & $\begin{array}{l}0,00 \\
(0,06)\end{array}$ & $\begin{array}{l}-0,00 \\
(0,02)\end{array}$ \\
\hline Freizeit: Sport & & & $\begin{array}{l}0,03 \\
(0,02)^{*}\end{array}$ & $\begin{array}{c}0,03 \\
(0,02)\end{array}$ & $\begin{array}{l}0,03 \\
(0,01)^{*}\end{array}$ & $\begin{array}{l}0,01 \\
(0,00)^{*}\end{array}$ \\
\hline Freizeit: Pfadfinder & & & $\begin{array}{l}-0,04 \\
(0,06)\end{array}$ & $\begin{array}{l}-0,03 \\
(0,06)\end{array}$ & $\begin{array}{l}-0,03 \\
(0,06)\end{array}$ & $\begin{array}{l}-0,01 \\
(0,02)\end{array}$ \\
\hline Konstante & $\begin{array}{l}-0,60 \\
(0,14)^{* * *}\end{array}$ & $\begin{array}{l}-0,18 \\
(0,16)\end{array}$ & $\begin{array}{l}-1,52 \\
(0,23)^{* * *}\end{array}$ & $\begin{array}{l}-1,65 \\
(0,26)^{* * *}\end{array}$ & $\begin{array}{l}-1,64 \\
(0,26)^{* * *}\end{array}$ & \\
\hline
\end{tabular}


Tab. 1: (Fortsetzung)

(Modell 1) (Modell 2) (Modell 3) (Modell 4) (Modell 5) MPE

(Modell 3)

\begin{tabular}{lllllll}
\hline $\mathrm{F}$ & 1,04 & 8,67 & 13,61 & 13,32 & 14,17 & 13,61 \\
$\mathrm{~N}$ & 933 & 933 & 933 & 933 & 933 & 933 \\
\hline
\end{tabular}

Survey Probit Regression (Standardfehler sind für die Clusterstichprobe bereinigt; Gewichtung für sampling probability)

Signifikanzniveaus: ${ }^{*} \mathrm{p}<0,05, * * \mathrm{p}<0,01, * * * \mathrm{p}<0,001$

zu wählen, als ihre mathematisch weniger begabten Kommilitonen (vgl. Marginaleffekt von plus 18 Prozentpunkten). Untersucht man den Distanzeffekt nur für die Gruppe der Maturanden mit den besten Mathematiknoten (Modell 5 in Tab. 1), so ist der Distanzeffekt nicht mehr signifikant von Null verschieden, d. h., sehr gute Maturanden lassen sich nicht aus Distanzgründen von einem Studium an der ETHZ abhalten. Auch dieser Befund scheint einem bekannten Muster zu entsprechen, wonach leistungsstarke Studierende in ihrer Hochschulwahl wesentlich unabhängiger vom Faktor Distanz sind (vgl. Avery und Hoxby 2004, S. 288). ${ }^{19}$ Diese Inelastizität bezüglich der Distanz bei guter schulischer Leistung kann mit dem in diesem Fall geringeren Investitionsrisiko der Bildungsentscheidung erklärt werden (vgl. Esser 2002, S. 269): Mit guten schulischen Fähigkeiten steigen die Erfolgswahrscheinlichkeit wie auch der zu erwartende Bildungsertrag, sodass sich die Wahl ETHZ trotz höherer Studienkosten immer noch lohnt.

\subsubsection{Wahl eines Studienfachs an einer lokalen Spartenuniversität (Rechtswissenschaften an der Universität Luzern)}

Die Auswertungen für das Einzugsgebiet Luzern (vgl. Tab. 2) bestätigen unsere eingangs formulierte Hypothese ebenfalls: Es kann über alle Spezifikationen festgestellt werden (inklusive der Robustness-Checks mit unterschiedlich definiertem Einzugsgebiet „LUlarge“ respektive „LU-small“ im Modell 4 bzw. 5), dass die geographische Nähe zur Universität Luzern die Wahrscheinlichkeit signifikant erhöht, Jurisprudenz als Studienfach zu wählen. Im Einzugsgebiet Luzern beträgt die Neigung für Rechtswissenschaften mehr als das Doppelte als in anderen Regionen. ${ }^{20}$ Nach Schicht (SES) differenziert betrachtet, zeigt sich, dass Maturanden aus der obersten sozioökonomischen Schicht (SES 3) sich generell eher für ein Studium in Rechtswissenschaften interessieren als jene aus der mittleren und der unteren Schicht (signifikanter Effekt SES 3 in den Modellen 3 bis 5). Die Interaktion mit der Distanzvariable (Modell 6) zeigt hier jedoch - genau wie im Fall der ETHZ -, dass die oberste sozioökonomische Schicht von der Distanz nicht signifikant beeinflusst wird $(\mathrm{F}[1 ; 40]=0,81, \mathrm{p}>0,1)$. Mit anderen Worten, Maturanden aus dieser Schicht weisen, auch wenn sie in der Nähe der Universität Luzern wohnen, keine größere Wahrscheinlichkeit auf, sich für Rechtswissenschaften zu entscheiden. Es sind wiederum die mittlere und die unterste sozioökonomische Schicht, deren Studienentscheid die Distanz offenbar signifikant beeinflusst. 
Tab. 2: Wahrscheinlichkeit der Wahl der Rechtswissenschaften unter Berücksichtigung des Einzugsgebiets der Universität Luzern (Probit-Regression)

(Modell 1) (Modell 2) (Modell 3) (Modell 4) (Modell 5) (Modell 6) MPE

(Modell 3)

\begin{tabular}{|c|c|c|c|c|c|c|c|}
\hline$\overline{L U \_d u m m y}$ & $\begin{array}{l}0,27 \\
(0,12)^{*}\end{array}$ & $\begin{array}{l}0,39 \\
(0,18)^{*}\end{array}$ & $\begin{array}{l}0,55 \\
(0,17)^{* *}\end{array}$ & & & $\begin{array}{l}0,30 \\
(0,11)^{*}\end{array}$ & $\begin{array}{l}0,10 \\
(0,04)^{*}\end{array}$ \\
\hline LU_large & & & & $\begin{array}{l}0,54 \\
(0,17)^{* *}\end{array}$ & & & \\
\hline LU_small & & & & & $\begin{array}{l}0,55 \\
(0,19)^{* *}\end{array}$ & & \\
\hline SES 1: tief (d) & $\begin{array}{l}0,11 \\
(0,14)\end{array}$ & $\begin{array}{l}0,13 \\
(0,14)\end{array}$ & $\begin{array}{l}0,16 \\
(0,15)\end{array}$ & $\begin{array}{l}0,16 \\
(0,15)\end{array}$ & $\begin{array}{l}0,16 \\
(0,14)\end{array}$ & $\begin{array}{l}-0,03 \\
(0,16)\end{array}$ & $\begin{array}{l}0,03 \\
(0,02)\end{array}$ \\
\hline SES 2: mittel (d) & Referenzk & ategorie & & & & & \\
\hline SES 3: hoch (d) & $\begin{array}{l}0,46 \\
(0,22)^{*}\end{array}$ & $\begin{array}{l}0,35 \\
(0,18)\end{array}$ & $\begin{array}{l}0,39 \\
(0,19)^{*}\end{array}$ & $\begin{array}{l}0,39 \\
(0,19)^{*}\end{array}$ & $\begin{array}{l}0,39 \\
(0,19)^{*}\end{array}$ & $\begin{array}{l}0,42 \\
(0,21)\end{array}$ & $\begin{array}{l}0,07 \\
(0,04)\end{array}$ \\
\hline $\begin{array}{l}\text { Interaktion SES } \\
1 * \mathrm{LU}\end{array}$ & & & & & & $\begin{array}{l}0,76 \\
(0,37)^{*}\end{array}$ & \\
\hline $\begin{array}{l}\text { Interaktion SES } \\
3 * \mathrm{LU}\end{array}$ & & & & & & $\begin{array}{l}-0,40 \\
(0,35)\end{array}$ & \\
\hline Alter (zentriert) & $\begin{array}{l}-0,05 \\
(0,10)\end{array}$ & $\begin{array}{l}-0,08 \\
(0,09)\end{array}$ & $\begin{array}{l}-0,10 \\
(0,10)\end{array}$ & $\begin{array}{l}-0,10 \\
(0,10)\end{array}$ & $\begin{array}{l}-0,10 \\
(0,10)\end{array}$ & $\begin{array}{l}-0,09 \\
(0,10)\end{array}$ & $\begin{array}{l}-0,01 \\
(0,02)\end{array}$ \\
\hline Frau (d) & $\begin{array}{l}0,30 \\
(0,12)^{*}\end{array}$ & $\begin{array}{l}0,17 \\
(0,13)\end{array}$ & $\begin{array}{l}0,21 \\
(0,14)\end{array}$ & $\begin{array}{l}0,21 \\
(0,14)\end{array}$ & $\begin{array}{l}0,22 \\
(0,14)\end{array}$ & $\begin{array}{c}0,24 \\
(0,15)\end{array}$ & $\begin{array}{l}0,03 \\
(0,02)\end{array}$ \\
\hline \multicolumn{8}{|c|}{ Fächerprofil am Gymnasium: } \\
\hline Sprachen (d) & & $\begin{array}{l}-0,02 \\
(0,22)\end{array}$ & $\begin{array}{l}0,06 \\
(0,23)\end{array}$ & $\begin{array}{l}0,06 \\
(0,23)\end{array}$ & $\begin{array}{l}0,06 \\
(0,23)\end{array}$ & $\begin{array}{l}0,05 \\
(0,22)\end{array}$ & $\begin{array}{l}0,01 \\
(0,03)\end{array}$ \\
\hline Math./Science (d) & & $\begin{array}{l}-0,95 \\
(0,29)^{* *}\end{array}$ & $\begin{array}{l}-0,60 \\
(0,34)\end{array}$ & $\begin{array}{l}-0,60 \\
(0,34)\end{array}$ & $\begin{array}{l}-0,60 \\
(0,34)\end{array}$ & $\begin{array}{l}-0,70 \\
(0,31)^{*}\end{array}$ & $\begin{array}{l}-0,07 \\
(0,03)^{*}\end{array}$ \\
\hline Wirtschaft/Recht (d & & Referenzk & ategorie & & & & \\
\hline Musisch (d) & & $\begin{array}{l}-0,51 \\
(0,24)^{*}\end{array}$ & $\begin{array}{l}-0,36 \\
(0,27)\end{array}$ & $\begin{array}{l}-0,35 \\
(0,27)\end{array}$ & $\begin{array}{l}-0,35 \\
(0,27)\end{array}$ & $\begin{array}{l}-0,32 \\
(0,25)\end{array}$ & $\begin{array}{l}-0,04 \\
(0,03)\end{array}$ \\
\hline $\begin{array}{l}\text { Note Math., } 4 . \\
\text { Quartil (d) }\end{array}$ & & $\begin{array}{l}-0,55 \\
(0,18)^{* *}\end{array}$ & $\begin{array}{l}-0,42 \\
(0,19)^{*}\end{array}$ & $\begin{array}{l}-0,42 \\
(0,19)^{*}\end{array}$ & $\begin{array}{l}-0,42 \\
(0,19)^{*}\end{array}$ & $\begin{array}{l}-0,40 \\
(0,19)^{*}\end{array}$ & $\begin{array}{l}-0,05 \\
(0,02)^{*}\end{array}$ \\
\hline $\begin{array}{l}\text { Wissenschafts- } \\
\text { orienterung }\end{array}$ & & & $\begin{array}{l}-0,26 \\
(0,09)^{* *}\end{array}$ & $\begin{array}{l}-0,25 \\
(0,09)^{* *}\end{array}$ & $\begin{array}{l}-0,26 \\
(0,09)^{* *}\end{array}$ & $\begin{array}{l}-0,27 \\
(0,10)^{* *}\end{array}$ & $\begin{array}{l}-0,04 \\
(0,02)^{*}\end{array}$ \\
\hline $\begin{array}{l}\text { Karriereorien- } \\
\text { tierung }\end{array}$ & & & $\begin{array}{l}0,41 \\
(0,08)^{* * *}\end{array}$ & $\begin{array}{l}0,41 \\
(0,08)^{* * *}\end{array}$ & $\begin{array}{l}0,42 \\
(0,08)^{* * *}\end{array}$ & $\begin{array}{l}0,44 \\
(0,07) * * *\end{array}$ & $\begin{array}{l}0,06 \\
(0,01)^{* * *}\end{array}$ \\
\hline Freizeit: Sport & & & $\begin{array}{l}-0,03 \\
(0,02)\end{array}$ & $\begin{array}{l}-0,03 \\
(0,02)\end{array}$ & $\begin{array}{l}-0,03 \\
(0,02)\end{array}$ & $\begin{array}{l}-0,03 \\
(0,02)\end{array}$ & $\begin{array}{l}-0,00 \\
(0,00)\end{array}$ \\
\hline Freizeit: Pfadfinde & & & $\begin{array}{l}-0,05 \\
(0,04)\end{array}$ & $\begin{array}{l}-0,05 \\
(0,04)\end{array}$ & $\begin{array}{l}-0,05 \\
(0,04)\end{array}$ & $\begin{array}{l}-0,05 \\
(0,04)\end{array}$ & $\begin{array}{l}-0,01 \\
(0,01)\end{array}$ \\
\hline Konstante & $\begin{array}{l}-1,49 \\
(0,15)^{* * *}\end{array}$ & $\begin{array}{l}-1,08 \\
(0,21)^{* * *}\end{array}$ & $\begin{array}{l}-1,27 \\
(0,24) * * *\end{array}$ & $\begin{array}{l}-1,26 \\
(0,24)^{* * *}\end{array}$ & $\begin{array}{l}-1,26 \\
(0,24)^{* * *}\end{array}$ & $\begin{array}{l}-1,24 \\
(0,23)^{* * *}\end{array}$ & \\
\hline
\end{tabular}


Tab. 2: (Fortsetzung)

\begin{tabular}{llllllll}
\hline \multicolumn{7}{c}{ (Modell 1) (Modell 2) (Modell 3) (Modell 4) (Modell 5) (Modell 6) MPE } \\
& \multicolumn{7}{c}{ (Modell 3) } \\
\hline $\mathrm{F}$ & 3,63 & 4,93 & 10,39 & 10,40 & 9,52 & 9,56 & 10,39 \\
$\mathrm{~N}$ & 933 & 933 & 933 & 933 & 933 & 933 & 933 \\
\hline
\end{tabular}

Survey Probit Regression (Standardfehler sind für die Clusterstichprobe bereinigt; Gewichtung für sampling probability)

Signifikanzniveau: $* \mathrm{p}<0,05, * * \mathrm{p}<0,01, * * * \mathrm{p}<0,001$

Der Zusammenhang zwischen schulischer Leistung und Studienfachwahl deutet auf einen negativen Zusammenhang zwischen Mathematiknoten und der Wahrscheinlichkeit, Jurisprudenz zu studieren. ${ }^{21}$ Interagiert man die Mathematiknoten mit der Distanzvariable, lässt sich kein signifikanter Distanzeffekt für Studierende mit guten Mathematiknoten mehr feststellen $(\mathrm{F}[1 ; 40]=0,06 ; \mathrm{p}>0,1)$. Auch hier gilt folglich: Sehr gute Maturanden lassen sich durch die Nähe zu einer bestimmten Universität in ihrer Studienfachwahl nicht einschränken. Dies bedeutet für die lokale Universität aber auch, dass sie ihre Studierenden vor allem aus dem Feld der sozioökonomisch weniger privilegierten und schulisch weniger exzellenten Maturanden rekrutieren muss, die im Durchschnitt auch stärkeren Distanzrestriktionen unterliegen.

\subsubsection{Wahl eines Studiengangs an einer pädagogischen Hochschule}

Auch die Anwendung unserer Analyse auf die Situation der pädagogischen Hochschulen (Tab. 3) bestätigt die eingangs formulierte Hypothese. Es lässt sich durchgängig ein Distanzeffekt nachweisen (vgl. komplettes Modell 4, Tab.3): Die Wahrscheinlichkeit, dass Maturanden ein Studium an einer pädagogischen Hochschule anstreben, steigt mit der Entfernung zur nächstgelegenen Universität. ${ }^{22}$ Das bedeutet, dass es an den Standorten mit einer pädagogischen Hochschule, aber keiner Universität (was am negativen Effekt der Variable Uni-Kanton in der Modell-Spezifikation 2, Tab.3, ersichtlich ist) zu einem angebotsinduzierten Nachfrageverhalten kommt.

Generell lässt sich sagen, dass - neben anderen Faktoren wie Geschlecht und Fächerprofil am Gymnasium sowie motivationaler Faktoren ${ }^{23}$ - das Studienfach Lehramt vor allem von den unteren sozioökonomischen Schichten gewählt wird; statushöhere Schichten haben eine geringere Wahrscheinlichkeit, ein PH-Studium zu wählen (vgl. die signifikant positiven Koeffizienten der Variable „SES 1“ sowie die negativen der Variable „SES 3“). Die Interaktion mit der Distanzvariablen weist hingegen einen signifikanten Effekt für die Mittelschicht nach (vgl. Koeffizient „Distanz Uni“"24 im Modell 5). Das bedeutet, dass es - ähnlich wie in den beiden anderen Fällen - die Mittelschicht ist, welche am stärksten auf den Faktor Distanz bei der Studienentscheidung reagiert. 
Tab. 3: Wahrscheinlichkeit Wahl eines Studiums an einer Pädagogischen Hochschule (ProbitRegression)

\begin{tabular}{|c|c|c|c|c|c|c|c|}
\hline \multirow[b]{2}{*}{ Distanz Uni } & \multicolumn{7}{|c|}{$\begin{array}{l}\text { (Modell 1) (Modell 2) (Modell 3) (Modell 4) (Modell 5) (Modell 6) MPE } \\
\text { (Mod. 4) }\end{array}$} \\
\hline & $\begin{array}{l}0,16 \\
(0,06)^{*}\end{array}$ & & $\begin{array}{l}0,17 \\
(0,06)^{* *}\end{array}$ & $\begin{array}{l}0,18 \\
(0,06)^{* *}\end{array}$ & $\begin{array}{l}0,20 \\
(0,08)^{*}\end{array}$ & $\begin{array}{l}0,18 \\
(0,06)^{* *}\end{array}$ & $\begin{array}{l}0,03 \\
(0,01)^{*}\end{array}$ \\
\hline Universiätskanton & & $\begin{array}{l}-0,18 \\
(0,09)^{+}\end{array}$ & & & & & \\
\hline SES 1: tief (d) & $\begin{array}{l}0,28 \\
(0,11)^{*}\end{array}$ & $\begin{array}{l}0,28 \\
(0,11)^{*}\end{array}$ & $\begin{array}{l}0,22 \\
(0,11)^{+}\end{array}$ & $\begin{array}{l}0,25 \\
(0,12)^{*}\end{array}$ & $\begin{array}{l}0,23 \\
(0,12)^{+}\end{array}$ & $\begin{array}{l}0,25 \\
(0,12)^{*}\end{array}$ & $\begin{array}{l}0,04 \\
(0,02)^{+}\end{array}$ \\
\hline SES 2: mittel (d) & \multicolumn{7}{|c|}{ Referenzkategorie } \\
\hline SES 3: hoch (d) & $\begin{array}{l}-0,29 \\
(0,16)^{+}\end{array}$ & $\begin{array}{l}-0,28 \\
(0,16)^{+}\end{array}$ & $\begin{array}{l}-0,21 \\
(0,19)\end{array}$ & $\begin{array}{l}-0,12 \\
(0,20)\end{array}$ & $\begin{array}{l}-0,10 \\
(0,19)\end{array}$ & $\begin{array}{l}-0,12 \\
(0,20)\end{array}$ & $\begin{array}{l}-0,01 \\
(0,02)\end{array}$ \\
\hline $\begin{array}{l}\text { Interaktion SES } \\
1 * \text { Distanz }\end{array}$ & & & & & $\begin{array}{l}-0,06 \\
(0,10)\end{array}$ & & \\
\hline $\begin{array}{l}\text { Interaktion SES } \\
3 * \text { Distanz }\end{array}$ & & & & & $\begin{array}{l}0,04 \\
(0,08)\end{array}$ & & \\
\hline Alter (zentriert) & & & $\begin{array}{l}0,12 \\
(0,09)\end{array}$ & $\begin{array}{l}0,08 \\
(0,08)\end{array}$ & $\begin{array}{l}0,08 \\
(0,08)\end{array}$ & $\begin{array}{l}0,08 \\
(0,08)\end{array}$ & $\begin{array}{l}0,01 \\
(0,01)\end{array}$ \\
\hline Frau (d) & & & $\begin{array}{l}1,18 \\
(0,14)^{* *}\end{array}$ & $\begin{array}{l}1,01 \\
(0,17)^{* *}\end{array}$ & $\begin{array}{l}1,01 \\
(0,17)^{* *}\end{array}$ & $\begin{array}{l}1,01 \\
(0,17)^{* *}\end{array}$ & $\begin{array}{l}0,06 \\
(0,02)^{* *}\end{array}$ \\
\hline \multicolumn{8}{|c|}{ Fächerprofil am Gymnasium: } \\
\hline Sprachen (d) & & & Referenzl & ategorie & & & \\
\hline Math./Science (d) & & & & $\begin{array}{l}-0,31 \\
(0,29)\end{array}$ & $\begin{array}{l}-0,32 \\
(0,29)\end{array}$ & $\begin{array}{l}-0,31 \\
(0,29)\end{array}$ & $\begin{array}{l}-0,04 \\
(0,02)\end{array}$ \\
\hline Wirtschaft/Recht (d) & & & & $\begin{array}{l}0,28 \\
(0,15)^{+}\end{array}$ & $\begin{array}{l}0,28 \\
(0,15)^{+}\end{array}$ & $\begin{array}{l}0,28 \\
(0,15)^{+}\end{array}$ & $\begin{array}{l}0,04 \\
(0,02)\end{array}$ \\
\hline Musisch (d) & & & & $\begin{array}{l}0,58 \\
(0,16)^{* *}\end{array}$ & $\begin{array}{l}0,58 \\
(0,16) * *\end{array}$ & $\begin{array}{l}0,58 \\
(0,16)^{* *}\end{array}$ & $\begin{array}{l}0,10 \\
(0,03)^{* *}\end{array}$ \\
\hline $\begin{array}{l}\text { Note Deutsch, } 90 . \\
\text { Perzentil (d) }\end{array}$ & & & & $\begin{array}{l}-0,72 \\
(0,21)^{* *}\end{array}$ & $\begin{array}{l}-0,72 \\
(0,21)^{* *}\end{array}$ & $\begin{array}{l}-0,73 \\
(0,22)^{* *}\end{array}$ & $\begin{array}{l}-0,05 \\
(0,02)^{* *}\end{array}$ \\
\hline $\begin{array}{l}\text { Note } \\
\text { Deutsch*Distanz }\end{array}$ & & & & & & $\begin{array}{l}-0,03 \\
(0,31)\end{array}$ & \\
\hline $\begin{array}{l}\text { Wissenschaftsorien- } \\
\text { tierung }\end{array}$ & & & & $\begin{array}{l}-0,24 \\
(0,05)^{* *}\end{array}$ & $\begin{array}{l}-0,24 \\
(0,05)^{* *}\end{array}$ & $\begin{array}{l}-0,24 \\
(0,05)^{* *}\end{array}$ & $\begin{array}{l}-0,03 \\
(0,01)^{* *}\end{array}$ \\
\hline Karriereorientierung & & & & $\begin{array}{l}-0,25 \\
(0,04)^{* *}\end{array}$ & $\begin{array}{l}-0,25 \\
(0,04)^{* *}\end{array}$ & $\begin{array}{l}-0,25 \\
(0,04)^{* *}\end{array}$ & $\begin{array}{l}-0,03 \\
(0,01)^{* *}\end{array}$ \\
\hline Freizeit: Sport & & & & $\begin{array}{l}-0,00 \\
(0,01)\end{array}$ & $\begin{array}{l}-0,00 \\
(0,01)\end{array}$ & $\begin{array}{l}-0,00 \\
(0,01)\end{array}$ & $\begin{array}{l}-0,00 \\
(0,01)\end{array}$ \\
\hline Freizeit: Pfadfinder & & & & $\begin{array}{l}0,06 \\
(0,04)^{+}\end{array}$ & $\begin{array}{l}0,06 \\
(0,04)^{+}\end{array}$ & $\begin{array}{l}0,06 \\
(0,04)^{+}\end{array}$ & $\begin{array}{l}0,01 \\
(0,01)\end{array}$ \\
\hline
\end{tabular}


Tab. 3: (Fortsetzung)

\begin{tabular}{llllllll}
\hline \multicolumn{7}{l}{ (Modell 1) (Modell 2) (Modell 3) (Modell 4) (Modell 5) (Modell 6) MPE } \\
(Mod. 4)
\end{tabular}

Survey Probit Regression (Standardfehler sind für die Clusterstichprobe bereinigt; Gewichtung für sampling probability)

Signifikanzniveau: ${ }^{+} \mathrm{p}<0,10,{ }^{*} \mathrm{p}<0,05, * * \mathrm{p}<0,01$

\section{Schlussfolgerungen}

Im vorliegenden Aufsatz wurde der Einfluss der Nähe zu einer Hochschule sowohl auf die Wahl der Hochschule als auch auf die Wahl eines bestimmten Studienfachs untersucht. Für die empirische Überprüfung dieser Fragen eignet sich das schweizerische Hochschulwesen besonders gut, weil Studierende in der Schweiz die Hochschule und das Studienfach frei wählen können und weil das Studium an schweizerischen Hochschulen mit mehr oder weniger einheitlich tiefen Studiengebühren verbunden ist. Mit anderen Worten, es kann nicht davon ausgegangen werden, dass das Studienverhalten stark durch Entscheidungen der Hochschulen selbst beeinflusst wird.

Unsere Untersuchung hat den empirischen Nachweis geliefert, dass die Distanz zur nächstgelegenen Hochschule in der Deutschschweiz Studienentscheide signifikant beeinflusst. Auf der einen Seite nimmt die Wahrscheinlichkeit, ein Fach zu studieren, welches nur an einer einzigen Hochschule (z. B. ETH Zürich) angeboten wird, mit der Distanz zu dieser Hochschule ab. Andererseits wächst die Wahrscheinlichkeit, ein ganz bestimmtes Fach zu studieren, wenn man in der Nähe einer Universität wohnt, welche ein eingeschränktes Fächerangebot mit Schwerpunkt auf diesem Fach aufweist (z. B. Luzern). Dieser Angebotseffekt lässt sich sogar bei einem spezifischen Hochschultyp zeigen: Die Nachfrage nach einem Studium an einer pädagogischen Hochschule steigt, je weiter man von einer universitären Hochschule entfernt wohnt.

Unterscheidet man die potenziellen Studierenden nach ihrer sozioökonomischen Herkunft, lässt sich weiter zeigen, dass Studierende aus sozioökonomisch privilegiertem Elternhaus in ihren Entscheidungen keinen Distanzrestriktionen unterworfen sind. Dies kann auch als Indiz dafür gewertet werden, dass dort, wo die Distanzeffekte das Studienverhalten signifikant beeinflussen, der Grund darin liegt, dass mit steigender Distanz zu einer Hochschule die tatsächlichen Kosten eines Studiums steigen. Dies trägt dazu bei, den hier verfolgen humankapitaltheoretischen Erklärungsansatz zu erhärten. Differenziert man weiter nach der schulischen Leistung, lässt sich feststellen, dass die besten Maturanden sich in ihren Studienwahlentscheidungen nicht durch die Distanz zur Hochschule beeinflussen lassen.

Für die Hochschulpolitik lassen diese Befunde drei Schlussfolgerungen zu: 
Erstens ist ein relativ großer Teil der potentiellen Studierenden erheblichen Distanzrestriktionen in der Wahl der Hochschule und des Studienfachs unterworfen. Dies kann zu einer Beschränkung sowohl der allokativen wie die produktiven Effizienz des Hochschulsystems führen. Die allokative Effizienz ist nicht gewährleistet, weil nicht alle Studierenden den Studienentscheid ihrer freien Wahl treffen können. Im Fall von Spartenuniversitäten (Luzern) lässt sich eine Tendenz zu angebotsinduzierter Nachfrage bezüglich der angebotenen Studienfächer feststellen. Dies gilt auch für den dritten Typus von Hochschulinstitutionen: die neu eingerichteten pädagogischen Hochschulen. Und die produktive Effizienz wird deshalb eingeschränkt, weil die Distanzrestriktionen auch den Wettbewerb zwischen den Hochschulen um Studierende vermindern. Je größer die Distanzrestriktionen der Studierenden sind, desto eher bilden sich bei den Anbietern lokale Monopole heraus.

Zweitens offenbart die Distanzrestriktion auch ein Equity-Problem, da nur die sozioökonomisch weniger privilegierten Studierenden in ihren Entscheidungen eingeschränkt werden. Das Equity-Problem wäre nur dann vernachlässigbar, wenn regional flächendeckend ein qualitativ und quantitativ identisches Hochschulangebot verfügbar wäre, was sicherlich nicht der Fall ist.

Schließlich wirken sich drittens die Distanzrestriktionen ganz unterschiedlich auf die einzelnen Hochschultypen aus. Die Hochschulen mit hoher Reputation (ähnlich den „selective colleges“ in den USA; vgl. Hoxby 2009) sind gemäß unseren Ergebnissen in der Lage, sich auch unter den potenziell distanzrestringierten Studierenden eine Nachfrage zu sichern, wenn diese zu den leistungsstärksten Studierenden gehören. Dies bedeutet aber gleichzeitig, dass die durchschnittliche Hochschule dann umso mehr nur noch unter den weniger mobilen Studierenden rekrutieren kann und entsprechend weniger selektiv sein kann.

Analog gilt für die pädagogischen Hochschulen bezüglich der Angebotsdichte ein Trade-off zwischen Qualität und Quantität: Je dezentraler die einzelnen pädagogischen Hochschulen angesiedelt sind und damit einer sehr begrenzten und lokalen Nachfrage nach Studienplätzen gegenüberstehen, desto eher müssen sie unter den weniger leistungsstarken Studierenden rekrutieren (vgl. Alm und Winters 2009). Dieser Tendenz könnte entsprechend dem Beispiel der ETHZ wahrscheinlich nur dann entgegengewirkt werden, wenn die Lehrkräfteausbildung zentral an einem Standort stattfände. Dabei müsste allerdings sichergestellt werden, dass eine solche Hochschule über eine hohe Reputation verfügt. So könnte, abgesehen vom ungelösten Equity-Problem, immerhin gewährleistet werden, dass die hochmotivierten und leistungsstarken Studierenden trotz Distanzrestriktionen ein solches Studium aufnähmen.

Danksagung: Die Autoren danken den drei anonymen Gutachtern sowie der Schriftleitung für ihre hilfreichen Kommentare. 


\section{Anmerkungen}

1 Der Vollständigkeit halber kann angefügt werden, dass der Entscheid, zu studieren oder nicht zu studieren, trotzdem empirisch geprüft wurde, wobei sich aber - wie erwartet - keine Distanzabhängigkeit zeigte.

2 In der Schweiz ergibt sich dieser sozialräumliche Einfluss auf die Wahrscheinlichkeit, ein Studium zu beginnen, weniger aufgrund unterschiedlicher Studierneigungen als vielmehr aufgrund unterschiedlicher Wahrscheinlichkeit, überhaupt eine Studierberechtigung (gymnasiale Maturität) zu erreichen, da die Maturitätsquoten in ländlichen Gebieten deutlich tiefer liegen als in städtischen Gebieten, in denen auch die Hochschulen zu finden sind (vgl. SKBF 2010).

3 Spieß und Wrohlich (2010) sprechen von „Transaktionskosten“, die mit zunehmender Distanz zur Universität ansteigen. Transaktionskosten sind Kosten, die nicht bei der Gütererstellung, sondern bei der Übertragung von einem Wirtschaftssubjekt zum anderen entstehen; m. a. W., Kosten, die durch die Benützung des Marktes entstehen, also bspw. Informations- und Beschaffungskosten.

4 Die kantonalen Universitäten sowie die ETH unterscheiden sich von den anderen Hochschultypen (Fachhochschulen und pädagogische Hochschulen) insbesondere dadurch, dass sie auch das Recht haben, Doktorate zu vergeben. Die Schulen der ersten Kategorie werden deshalb in diesem Artikel auch als universitäre oder akademische Hochschulen bezeichnet.

5 Die $90 \%$ beziehen sich auf die gesamte Schweiz. In unserer Stichprobe, welche sich nur auf die Deutschschweiz bezog, lag der Anteil der Maturanden, die gar kein Studium ergreifen wollten, bei $5 \%$. Allerdings handelt es sich hier um Absichtserklärungen, während die offizielle Statistik die erfolgten Studieneintritte erfasst.

6 Im Academic Ranking of World Universities (Shanghai Ranking) belegte die ETH Zürich 2009 Platz 23 und galt somit als die viertbeste Universität außerhalb der USA.

7 Die Universität Lugano, welche in der italienischsprachigen Schweiz liegt, ist für unsere Analyse von geringerem Interesse, da sie von Deutschschweizer Maturanden praktisch nicht besucht wird.

8 Die Fragestellung, ob das regionale Angebot die Studienfachwahl beeinflusst, ließe sich auch anhand der älteren Universität St. Gallen untersuchen. In diesem Fall zeigt sich zwar auch eine stärkere Verbreitung der Studienfachpräferenz Wirtschaft bei Maturanden, deren nächstgelegene Universität die Universität St. Gallen ist (19\% verglichen mit 14\% im Durchschnitt aller Maturanden). Dieser Unterschied ist allerdings statistisch nicht signifikant, was auch an der geringen Zahl der Beobachtungen liegen dürfte.

9 Studiengebühren ETHZ: 1288 Fr./Jahr; Universität Luzern: 1570 Fr./Jahr.

10 Um diesen Betrag durch Erwerbsarbeit finanzieren zu können, muss ein Studierender einer dauernden bezahlten Arbeit im Ausmaß von rund 20\% nachgehen, was gerade im Zuge der Bologna-Reform bei einzelnen Studienfächern mit rigiden Studienplänen schwierig geworden ist.

11 Die Stipendienbezügerquote schwankt erheblich zwischen den einzelnen Kantonen. Der Anteil an Hochschulstudierenden, die Stipendien erhalten, reicht von weniger als $10 \%$ in den Kantonen Zürich und Bern bis zu 30\% und mehr in den Kantonen Wallis, Graubünden oder Jura (vgl. BFS 2008).

12 Die meisten vergleichbaren Analysen sind regional eingeschränkt (bspw. ein US-Bundesstaat, eine kanadische Provinz). Unsere repräsentative Datenbasis ist zwar auf den deutschsprachigen Landesteil eingeschränkt, umfasst aber eine größere Anzahl Kantone mit unterschiedlichem Hochschulangebot und weist damit in der für uns interessierenden Frage eine genügend grosse Varianz auf. 
13 Respondquote: 0,88. Die Non-responses rührten mehrheitlich daher, dass einzelne Schüler zum Zeitpunkt der Befragung nicht in der Klasse anwesend waren (anderer Unterricht, Krankheit usw.), also nicht wegen einer Verweigerung der Befragung. Eine klassische Non-responseAnalyse wurde deshalb nicht durchgeführt. Einzelne Klassen mit einer Responsequote von unter 0,66 sowie solche, bei denen infolge systematischer Absenzen von einer verzerrten Befragungsteilnahme ausgegangen werden musste, wurden aus der Stichprobe ausgeschlossen.

14 Die Berechnungen erfolgten mittels der elektronischen Fahrzeitberechnung der Schweizerischen Bundesbahnen (SBB).

15 Dabei handelt es sich wie beim Logit-Modell um ein nichtlineares Wahrscheinlichkeitsmodell, wobei hier aber die kumulierte Verteilungsfunktion standardnormal verteilt ist.

16 Weil der Koeffizient $(\beta \mathrm{x})$ innerhalb der kumulativen Normalverteilungsfunktion erscheint $\operatorname{Pr}(\mathrm{y}=1)=\mathrm{F}(\alpha+\beta \mathrm{x})$, ist der Marginaleffekt im Unterschied zu einer linearen Regression nicht einfach durch den Beta-Koeffizienten gegeben, sondern muss von der kumulativen Verteilungsfunktion abgeleitet werden. Damit hängt der Effekt einer kleinen Änderung von $\mathrm{x}$ auf $\operatorname{Pr}(\mathrm{y}=1)$ vom Niveau aller erklärenden Variablen ab (Wooldridge 2003; Winkelmann und Boes 2006). Die Marginaleffekte im Probitmodell können dann wie folgt dargestellt werden: $M P E=\frac{\partial P\left(y_{i}=1\right)}{\partial x_{i l}}$.

17 Alle Regressionen wurden mit dem STATA Survey-Command (inkl. Gewichtungsfaktoren für die Stichprobenwahrscheinlichkeit) geschätzt, da es sich eine Clusterstichprobe handelt. Bei diesem Korrekturverfahren wird die Varianz eines Schätzers in der komplexen Stichprobe ins Verhältnis zur Varianz eines Schätzers in einer einfachen Zufallsstichprobe gesetzt.

18 Die Marginaleffekte zeigen je nach gewähltem Referenzindividuum etwa eine Abnahme um ein Drittel der prognostizierten Wahrscheinlichkeit für ein ETH-Studium.

19 Auch sind Hochschulinstitutionen mit einer sehr guten Forschungsreputation insgesamt gegen negative Distanzeffekte eher geschützt, wie die Analyse von Alm und Winters (2009) zeigt, die nachweisen, dass die Distanzelastizität der Studiennachfrage bei den zweijährigen Colleges am grössten ist, während bei den Forschungsuniversitäten die Nachfrage nach Studienplätzen praktisch distanzunabhängig ist.

20 Die Marginaleffekte zeigen je nach gewähltem Referenzindividuum für das Einzugsgebiet der Universität Luzern rund eine Verdoppelung der prognostizierten Wahrscheinlichkeit, Rechtswissenschaft zu studieren.

21 Analysiert man den Zusammenhang zwischen den schulischen Noten in der Muttersprache (Deutsch) und der Wahrscheinlichkeit, Jurisprudenz zu studieren, findet man einen positiven Effekt. Im Hinblick auf das Fach Deutsch sind es also die besseren Maturanden, die sich für das Studium der Rechtswissenschaften entscheiden.

22 Der Effekt ist relativ stark: Nimmt die Distanz zur nächsten Universität um eine Standardabweichung zu, so erhöht sich die prognostizierte Wahrscheinlichkeit, ein PH-Studium anzustreben um knapp die Hälfte (Referenzindividuum: Frau, mittlere SES-Schicht, musisches Profil mit durchschnittlichen Ausprägungen in den anderen Variablen).

23 Diese teilweise ebenfalls signifikanten Einflussgrößen, haben im vorliegenden Untersuchungsmodell primär die Funktion von Kontrollvariablen; sie stellen sicher, dass bei der Analyse des Distanzeffekts nicht etwa andere relevante Faktoren unberücksichtigt bleiben. Auf ihre Bedeutung im Bezug auf die Analyse der Studienwahl Lehramt wird in einer anderen Arbeit detailliert eingegangen (vgl. Denzler und Wolter 2009, S. 432).

24 Der Koeffizient für die Interaktion mit der mittleren SES-Schicht (Referenzkategorie) entspricht der Variable „Distanz Uni“: 0,20 (0,80). 


\section{Anhang}

Erläuterungen zu den Variablen

Distanz

Distanz zur ETHZ

Universitätskanton

LU_dummy

LU_small

LU_large

Alter (zentriert)

SES 1: tief

SES 2: mittel

SES 3: hoch

Sprachen

Math./Science

Wirtschaft/Recht

Musisch

Note Math., 4. Quartil

Note Deutsch, 90. Perz.

Wissenschaftsorienung

Karriereorientierung

Sport

Pfadfinder
Distanz Wohnort-Universität (standardisiert) in Std. öffentlicher Verkehr Distanz Wohnort-ETH (standardisiert) in Std. öffentlicher Verkehr kantonales Hochschulangebot $(0=$ keine HS, $1=$ Spartenuniversität, $2=$ Volluniversität)

Distanz Wohnort-Universität Luzern ist kürzer als zur nächst größeren Universität (Dummy)

Distanz Wohnort-Universität Luzern ist mindestens 10 Minuten kürzer als zur nächst größeren Universität (Dummy)

Distanz Wohnort-Universität Luzern ist höchstens 10 Minuten kürzer als zur nächst größeren Universität (Dummy)

Alter (zentriert)

sozioökonomischer Status: unteres Drittel (Dummy)

sozioökonomischer Status: mittleres Drittel (Dummy)

sozioökonomischer Status: oberes Drittel (Dummy)

Fächerprofil am Gymnasium: alte/neue Sprachen (Dummy)

Fächerprofil am Gymnasium: Mathematik/Naturwissenschaften (Dummy)

Fächerprofil am Gymnasium: Wirtschaft/Recht (Dummy)

Fächerprofil am Gymnasium: Musik/Gestalten; Pädagogik/Psychologie (Dummy)

Note Mathematik, oberstes Quartil (Dummy)

Note Deutsch, oberstes Dezil (Dummy)

Studienwahlmotive: Interesse für Wissenschaft und wissenschaftliches Arbeiten (Skala)

Studienwahlmotive: berufliche Karriere (Skala)

Freizeitbeschäftigung: Sport in Std. pro Woche

Freizeitbeschäftigung: Pfadfinder in Std. pro Woche

\section{Sicht-Variable (SES)}

Zur Bestimmung der Schichtzugehörigkeit wurde eine Variable gebildet, welche anhand der Angaben zum höchsten Bildungsabschluss sowie zur höchsten beruflichen Stellung mindestens eines Elternteil der befragen Maturanden eine Einschätzung der sozioökonomischen Stellung (SES) vornimmt. In den Regressionen kamen schließlich nur drei Schichtkategorien (niederer, mittlerer und hoher SES) zur Anwendung, weil die untersten zwei Schichten aufgrund geringer Fallzahlen zusammengefasst wurden. Diese ungleiche Verteilung hängt v.a. mit der sozialen Selektivität der Schüler am Gymnasium zusammen.

Für die Analysen wurden aufgrund der geringen Fallzahlen der Schicht IV die obersten beiden Schichten zusammengefasst. Damit wird nur noch zwischen SES-Schicht tief, mittel und hoch unterschieden. 


\begin{tabular}{|c|c|c|c|c|c|c|c|}
\hline & \multirow[b]{2}{*}{$\begin{array}{c}\text { keine } \\
\text { nachobligatorische } \\
\text { Ausbildung }\end{array}$} & \multicolumn{2}{|c|}{$\begin{array}{l}\text { Sekundarstufe } \\
\end{array}$} & \multicolumn{4}{|c|}{ Tertiärbildung } \\
\hline & & Berufsbildung & $\begin{array}{l}\text { Allgemeinbildung } \\
\text { (Maturitätsschule, } \\
\text { DMS, Seminar) }\end{array}$ & $\begin{array}{l}\text { Lehrerbildung } \\
\text { nachmaturitär }\end{array}$ & $\begin{array}{c}\begin{array}{c}\text { Fachhochschule/ } \\
\text { höhhere } \\
\text { Berufsbildung }\end{array}\end{array}$ & $\begin{array}{l}\text { Hochschule } \\
\text { (UH,ETH) }\end{array}$ & $\begin{array}{l}\text { Hochschule: } \\
\text { Promotion }\end{array}$ \\
\hline $\begin{array}{l}\text { Arbeitnehmer im } \\
\text { oberen Kader } \\
\text { (Direktor/Chef- } \\
\text { beamter) }\end{array}$ & & & & & & & \\
\hline $\begin{array}{l}\text { selbständig mit } \\
\text { eigenen } \\
\text { Angestellten }\end{array}$ & & & & & & & \\
\hline $\begin{array}{l}\text { Arbeitnehmer im } \\
\text { mittleren Kader }\end{array}$ & & & & & & & \\
\hline $\begin{array}{l}\text { selbständig ohne } \\
\text { Angestellte }\end{array}$ & & & & & & & \\
\hline Angestellter & & & & & & & \\
\hline & & I niedrig & & III gehoben & & & \\
\hline & & II mittel & & IV hoch & & & \\
\hline
\end{tabular}

\section{Literatur}

Alm, J., \& Winters, J. V. (2009). Distance and intrastate college student migration. Economics of Education Review, 28(6), 728-738.

Avery, C., \& Hoxby, C. (2004). Do and should financial aid packages affect students' college choices? In C. M. Hoxby (Hrsg.), A NBER conference report. College choices. The economics of where to go, when to go, and how to pay for it (S.239-299). Chicago: University of Chicago Press.

BFS (Bundesamt für Statistik). (2005). Soziale Lage der Studierenden in der Schweiz 2005: Erste Ergebnisse der Studierendenbefragung an den Hochschulen. Neuenburg: BFS.

BFS (Bundesamt für Statistik). (2007). Studien- und Lebensbedingungen an den Schweizer Hochschulen: Hauptbericht der Studie zur sozialen Lage der Studierenden 2005. Neuenburg: BFS.

BFS (Bundesamt für Statistik). (2008). Die soziale Dimension an den Hochschulen: Die Schweiz im europäischen Vergleich. Neuenburg: BFS.

BFS (Bundesamt für Statistik). (2009). Studienfachwahl und Hochschulwahl: Motivationale Aspekte. Neuenburg: BFS.

Chau, D. (2004). The effects of local colleges on the quality of college attended. Economics of Education Review, 23(2), 249-257.

Coradi Vellacott, M. (2007). Bildungschancen Jugendlicher in der Schweiz: Eine Untersuchung familiärer, schulischer und sozial-räumlicher Einflüsse auf Leistungsunterschiede am Ende der obligatorischen Schulzeit. Zürich: Rüegger.

Currie, J., \&. Moretti, E. (2003). Mother's education and the intergenerational transmission of human capital: Evidence from college openings. Quarterly Journal of Economics, 118(4), $1495-1532$.

Denzler, S., \& Wolter, S. C. (2009). Sorting into teacher education: How the institutional seting matters. Cambridge Journal of Education, 39(4), 423-441.

Drewes, T., \& Michael, C. (2006). How do students choose a university? An analysis of applications to universities in Ontario, Canada. Research in Higher Education, 47(7), 781-800.

Esser, H. (2002). Situationslogik und Handeln. Soziologie: Spezielle Grundlagen (Bd. 1). Frankfurt a. M.: Campus. 
Fabel, O., Lehmann, E., \& Warning, S. (2002). Der relative Vorteil deutscher wirtschaftswissenschaftlicher Fachbereiche im Wettbewerb um studentischen Zuspruch: Qualität des Studiengangs oder des Studienortes. Zeitschrift für betriebswirtschaftliche Forschung, 54, 509-526.

Frenette, M. (2006). Too far to go on? Distance to school and university participation. Education Economics, 14(1), 31-58.

Frenette, M. (2009). Do universities benefit local youth? Evidence from the creation of new universities. Economics of Education Review, 28(3), 378-328.

Griffith, A. L., \& Rothstein, D. S. (2009). Can't get there from here: The decision to apply to a selective college. Economics of Education Review, 28(5), 620-628.

Heinemann, K., \& Horch, H.-D. (1981). Soziologie der Sportorganisation. Sportwissenschaft, $11(2), 123-150$.

Hoxby, C. (2009). The changing selectivity of American colleges. Journal of Economic Perspectives, 23(4), 95-118.

Kesselring, H.-C. (1979). Kommunaler Finanzausgleich und Regionalpolitik. Grundlagen und Systematik: Eine empirische Untersuchung am Beispiel des Kantons Zürich. Zürich: Rüegger.

Kyung, W. (1996). In-migration of college students to the state of New York. Journal of Higher Education, 67(3), 349-358.

Long, B. T. (2004). How have college decisions changed over time? An application of the conditional logistic choice model. Journal of Econometrics, 121(1-2), 271-296.

McHugh, R., \& Morgan, J. N. (1984). The determinants of interstate student migration: A place-toplace analysis. Economics of Education Review, 3(4), 269-278.

Mechtenberg, L. (2005). Wettbewerb um kluge Köpfe: Die Länder Europas im Bologna-Prozess (WZB-Mitteilungen, No. 107). Berlin: WZB.

Mixon, F. G. (1992). Factors affecting college student migration across states. International Journal of Manpower, 13(1), 25-32.

Mixon, F. G., \& Hsing, Y. (1994). College student migration and human capital theory: A research note. Education Economics, 2(1), 65-73.

OECD. (Hrsg.). (1998). Redefining tertiary education. Paris: OECD.

Sà, C., Florax, R. J., \& Rietveld, P. (2004). Determinants of the regional demand for higher education in the Netherlands: A gravity model approach. Regional Studies, 38(4), 375-392.

Salvi, M., Schellenbauer, P., \& Schmidt, H. (2004). Preise, Mieten und Renditen: Der Immobilienmarkt transparent gemacht. Zürich: ZKB.

SBF (Staatssekretariat für Bildung und Forschung). (2004). Bericht über die Neuordnung der schweizerischen Hochschullandschaft. Bern: SBF.

SKBF (Schweizerische Koordinationsstelle für Bildungsforschung). (2010). Bildungsbericht Schweiz 2010. Aarau: SKBF.

Spieß, C. K., \& Wrohlich, K. (2010). Does distance determine who attends a university in Germany? Economics of Education Review, 29(3), 470-479.

Tinto, V. (1973). College proximity and rates of college attendance. American Educational Research Journal, 10(4), 277-293.

Tuckman, H. P. (1970). Determinants of college student migration. Southern Economic Journal, $37(2), 184-189$.

Turley, R. N. L. (2009). College proximity: Mapping access to opportunity. Sociology of Education, 82(2), 126-146.

Winkelmann, R., \& Boes, S. (2006). Analysis of microdata. Berlin: Springer.

Wooldridge, J. M. (2003). Introductory econometrics. A modern approach. Mason: South-Western. 